\title{
Development of a Nomogram for Moderate-to-Severe Bronchopulmonary Dysplasia or Death: Role of N-Terminal Pro-brain Natriuretic Peptide as a Biomarker
}

\section{OPEN ACCESS}

Edited by:

Bülent Taner Karadag,

Marmara University, Turkey

Reviewed by:

Hidehiko Nakanishi,

Kitasato University, Japan

Brian Sims,

University of Alabama at Birmingham,

United States

Vanajakshamma Velam,

Sri Venkateswara Institute of Medical

Sciences, India

${ }^{*}$ Correspondence:

Qian Zhang

zhangqian629@zzu.edu.cn

tThese authors have contributed equally to this work and share first

authorship

Specialty section:

This article was submitted to

Pediatric Pulmonology,

a section of the journal

Frontiers in Pediatrics

Received: 18 June 2021 Accepted: 02 August 2021

Published: 23 August 2021

Citation:

Song M, Lei M, Luo C, Shi Z, Cheng X, Ding W, Cao W, Zhang J, Ge J, Wang M, Xia P, Mao F, Wang L and Zhang $Q$ (2021) Development of a

Nomogram for Moderate-to-Severe

Bronchopulmonary Dysplasia or Death: Role of N-Terminal Pro-brain Natriuretic Peptide as a Biomarker.

Front. Pediatr. 9:727362.

doi: 10.3389/fped.2021.727362
Min Song ${ }^{1+}$, Mengyuan Lei ${ }^{2 \dagger}$, Chenghan Luo ${ }^{3}$, Zanyang Shi ${ }^{1}$, Xinru Cheng ${ }^{1}$, Wenqian Ding ${ }^{1}$, Wenjun Cao ${ }^{1}$, Jingdi Zhang ${ }^{1}$, Jian Ge ${ }^{1}$, Mengmeng Wang ${ }^{1}$, Peige Xia ${ }^{1}$, Fengxia Mao ${ }^{1}$, Li Wang ${ }^{1}$ and Qian Zhang ${ }^{1 *}$

${ }^{1}$ Neonatal Intensive Care Unit, The First Affiliated Hospital of Zhengzhou University, Zhengzhou, China, ${ }^{2}$ Health Care Department, The First Affiliated Hospital of Zhengzhou University, Zhengzhou, China, ${ }^{3}$ Orthopeadics Department, The First Affiliated Hospital of Zhengzhou University, Zhengzhou, China

Objectives: This study aimed to explore the clinical value of $\mathrm{N}$-terminal pro-brain natriuretic peptide (NT-proBNP) in predicting moderate-to-severe bronchopulmonary dysplasia (BPD)/death, and to establish an effective clinical predictive nomogram.

Methods: We retrospectively analyzed very low birth weight infants (VLBWs) with gestational age $\leq 32$ weeks. The NT-proBNP values were determined on the 1st, 3rd, 7th, 14th, 21st, and 28th days after birth. The correlation between NT-proBNP level and moderate-to-severe BPD/death was evaluated. Receiver operating characteristic (ROC) curve analysis was used to evaluate the prediction ability. Then, we used multivariable logistic regression to build the prediction model and nomogram, and calibration of the model was assessed by calibration curve.

Results: In total, 556 VLBWs were involved, among whom 229 developed BPD (mild: $n=109$; moderate: $n=68$; severe: $n=52$ ) and 18 died. The NT-proBNP level in the moderate-to-severe BPD/death group was significantly higher than that in the no-tomild BPD group from the 3rd to 28th day $(P<0.001)$. When the natural logarithm of the serum NT-ProBNP level increased by 1 unit at day 7 ( \pm 2 days) of life, the risk of moderate and severe BPD/death was the highest (OR = 3.753; 95\% Cl: 2.984 4.720), and ROC analysis identified an optimal cutoff point of $3360 \mathrm{ng} / \mathrm{L}$ (sensitivity: 80.0\%; specificity: 86.2\%; AUC: 0.861). After adjusting for confounding factors, the level of NT-proBNP at day 7 ( \pm 2 days) of life still had important predictive value for the development of moderate-to-severe BPD/death, significantly improving the predictive ability of the model.

Conclusion: The level of NT-proBNP at day 7 ( \pm 2 days) of life can be used as an early promising biomarker for VLBWs to develop moderate-to-severe BPD/death. We constructed an early predictive nomogram to help clinicians identify high-risk populations.

Keywords: bronchopulmonary dysplasia, $\mathrm{N}$-terminal pro-brain natriuretic peptide, biomarkers, nomogram, preterm infants 


\section{INTRODUCTION}

Bronchopulmonary dysplasia (BPD) is a serious chronic pulmonary morbidity in childhood and is one of the most common and serious sequelae of prematurity (1). The incidence of BPD in very low birth weight infants (VLBWs) is between 11 and 50\%, which is negatively correlated with gestational age (GA) and birth weight (BW) and varies considerably with different diagnostic criteria (2-4). With the improvement of neonatal care techniques, more premature infants, particularly VLBWs, can survive, leading to an increase in the incidence of BPD (5). Compared with patients with no-to-mild BPD, moderate-to-severe BPD affects survival and neurodevelopment, hospitalization is longer, mortality is higher, and it may be accompanied by a heavy life burden $(6,7)$. Presently, the evaluation time of BPD classification is 36 weeks postnatal menstrual age (PMA) or discharge to home, a time that is late and difficult for early intervention (8). Therefore, identifying predictors that can identify high-risk infants with moderate-tosevere BPD early is a critical research field.

$\mathrm{N}$-terminal pro-brain natriuretic peptide (NT-proBNP) is a low-molecular-weight peptide $(8.5 \mathrm{kDa})$ secreted by cardiomyocytes in response to volume overload (9). Compared with brain-type natriuretic peptide (BNP), NT-proBNP has a more stable chemical structure in vitro and can remain stable in blood samples for at least $72 \mathrm{~h}$ (10). Sellmer et al. (11) reported that NT-proBNP levels on day 3 were associated with BPD or death in a study of 183 premature infants whose GA was $<32$ weeks. In another small study ( $n=51)$ of GA $<30$ weeks, Harris et al. (12) found that the level of NT-proBNP at 10 days after birth was valuable in predicting severe BPD. Additionally, Khan et al. (13) showed that the NT-proBNP levels on the 28th day after birth were moderately predictive of the severity of BPD in a prospective study. These studies all measured the NT-proBNP levels on a particular day, but the postnatal NT-proBNP levels in newborns are a dynamic process. Our study had a larger sample size and dynamically measured NT-proBNP levels within 28 days of birth, features that can better evaluate the value of the serum NT-proBNP levels as an early biomarker of BPD severity or death. Thus, a convenient and effective clinical predictive model including clinical features and biochemical indicators can be established that may help high-risk children obtain effective individualized treatment in the early stage to avoid the occurrence of moderate-to-severe $\mathrm{BPD} /$ death as much as possible.

\section{MATERIALS AND METHODS}

\section{Patients and Study Design}

This retrospective study included premature infants delivered at the First Affiliated Hospital of Zhengzhou University and admitted to the Neonatal Intensive Care Unit (NICU) from November 01, 2016 to February 28, 2021 with GA $\leq 32$ weeks and $\mathrm{BW} \leq 1,500 \mathrm{~g}$. Infants with congenital metabolic defects or malformations, complex congenital heart disease [except patent foramen ovale (PFO) or patent ductus arteriosus (PDA)], severe renal insufficiency, death in the first week of life and incomplete general data were excluded. This study was approved by the Medical Ethics Committee of the First Affiliated Hospital of Zhengzhou University (Ethical code: 2019-KY-95).

\section{Data Collection and Definitions}

The data were collected from the maternal and infant medical records and included prenatal factors such as maternal age, hypertensive disorder complicating pregnancy (HDCP), gestational diabetes mellitus (GDM), hypothyroidism with pregnancy, the use of prenatal steroids, premature rupture of membranes (PROM), fetal distress and intrauterine growth restriction (IUGR), perinatal factors such as GA, BW, sex, assisted reproductive technology, mode of delivery, 1- and 5min Apgar scores, neonatal respiratory distress syndrome (RDS), hemodynamically significant patent ductus arteriosus (HsPDA) and pulmonary hypertension (PH). At the same time, the use of alveolar surfactant and caffeine and the time of mechanical ventilation (MV) in the first week of life were recorded. According to the definition of NICHD, the diagnostic criterion of BPD is that patients receive oxygen therapy with an oxygen concentration $>21 \%$ for at least 28 days, and are divided into no, mild, moderate or severe BPD groups according to the mode of respiratory support and concentration of supplementary oxygen at 36 weeks PMA (14).

Some perinatal risk factors in this paper were defined as follows: HDCP included preeclampsia, eclampsia, and gestational and chronic hypertension, which was defined as systolic blood pressure $\geq 140 \mathrm{mmHg}$ or $\geq 90 \mathrm{mmHg}$ during pregnancy (15). Hypothyroidism with pregnancy was defined as the level of serum thyrotropin exceeding the upper limit of the reference range and level of serum-free thyroxine 4 lower than the lower limit of the reference range during pregnancy (16). Fetal distress was clinically defined as the presence of long-term deceleration, repetitive moderate-to-severe variable deceleration or repetitive late deceleration (17). IUGR was defined as an estimated fetal weight or abdominal circumference less than the 10th percentile of GA (18). RDS was defined as a clinical symptom of early neonatal respiratory distress with consistent chest radiologic features and requiring oxygen supplementation within $24 \mathrm{~h}$ after birth to maintain a saturation of more than $85 \%$ (19). HsPDA was defined as the ductal diameter exceeds $1.5 \mathrm{~mm}$ plus echocardiography at 7 days ( \pm 2 days) after birth shows predominant left to right flow, and need for ventilator support with symptoms and signs suggestive of symptomatic PDA including respiratory deterioration, cardiac murmur, precordial hyperactivity, oliguria, hypotension and widening pulse pressure (20). Early PH was assessed by measuring tricuspid regurgitation (TR) in echocardiography at 7 days ( \pm 2 days) and calculating right ventricular systolic blood pressure (RVSP) using the modified Bernoulli equation described by Skinner et al. (21).

\section{NT-proBNP Measurement and Collection}

In the management of the NICU, our center attaches considerable importance to the monitoring of plasma NT-proBNP levels and cardiac function. For high-risk infants, the NT-proBNP levels were routinely measured on the 1st, 3rd, 7th, 14th and 28th days after birth. A small amount of venous blood samples was 
collected and centrifuged in a heparin lithium tube for $5 \mathrm{~min}$ (centrifugal radius $=6 \mathrm{~cm}, 3,500 \mathrm{r} / \mathrm{min}$ ). Next, the samples were immediately detected using a fully automatic electrochemical immunoluminescence analyzer (Cobas 8000 Roche Elecsys 602; Roche, Switzerland) according to the instruction manual. The detection range of the concentration was $5-35,000 \mathrm{ng} / \mathrm{L}$, and the coefficient of variation was $0.5 \%$.

The serum NT-proBNP levels measured on the 1st, 3rd, 7th, 14th, 21st and 28th days after birth were collected, and the complete data on the 1st and 3rd days were collected. If the data collected on the 7th and subsequent days were not accurately measured at the sampling time, the measured values 2 days before or after the sampling time were approximately replaced. If multiple samples were taken at the same sampling time, the average NT-proBNP value was selected. The NT-proBNP value higher than the quantitative upper limit $(>35,000 \mathrm{ng} / \mathrm{L})$ was changed to the fictive value of $35,010 \mathrm{ng} / \mathrm{L}$ in this study. On the day of blood collection, we also collected data on the types of oxygen supplementation and respiratory support.

\section{Sample Size Estimation}

In this study, 26 variables were analyzed by univariate analysis, and 14 variables were included in multivariate logistic regression. For binary endpoints, the rule of 10-20 events per variable (EPV) is generally followed to prevent overfitting (22). According to the principle of 20 EPVs per variable, this study required at least 280 EPVs. Our study included 556 preterm infants who met the criteria, so the sample size was sufficient.

\section{Statistical Analysis}

Statistical analysis was performed using $\mathrm{R}$ software (Version 3.6.3; https://www.R-project.org). The quantitative data were tested for normality using the Shapiro-Wilk test. All the quantitative data in the study showed normal distribution. These data were expressed as medians (upper quartile, lower quartile) and were compared with the Mann-Whitney $U$ test. The qualitative data were expressed as a percentage (\%) and compared by $\chi 2$ test. Natural logarithmic transformation was used to improve the model fitting of NT-proBNP measurements. Univariate logistic regression analysis was used to evaluate the contribution of the NT-proBNP levels to the development of moderate-to-severe $\mathrm{BPD} /$ death. Receiver operating characteristic (ROC) curve analysis was used to evaluate the prediction ability, and the Jordan index was used to calculate the best cutoff point. Multivariate logistic regression was used to determine the final model, and the area under the curve (AUC) was used to evaluate whether the prediction ability of the model was improved in the presence of biomarkers. The nomogram of the model was established and verified internally using 1,000 bootstrap analyses. A calibration curve was plotted to evaluate the performance of the nomogram. Differences were considered statistically significant at $P<0.05$.

\section{RESULTS}

During the study period, 581 premature infants with $\mathrm{GA} \leq$ 32 weeks and BW $\leq 1500 \mathrm{~g}$ were admitted to the NICU of the First Affiliated Hospital of Zhengzhou University. Among those patients, 12 were excluded because of congenital metabolic defects, complex heart disease or severe renal insufficiency, five were not included because of death in the first week of life, and eight were excluded because of incomplete general data. Finally, 556 premature infants were included in the study. Among the participants, 82 participants were premature infants with GA $\leq$ 28 weeks. Additionally, 229 infants met the diagnostic criteria of BPD, including 109 mild, 68 moderate and 52 severe infants, and 18 died between 7 days after birth and 36 weeks PMA. We divided all the participants into two groups. The first group included infants with no-to-mild BPD $(n=418)$, and the second group included infants with moderate-to-severe BPD/death $(n=138)$. Compared with group 1, the patients in group 2 had more unfavorable baseline characteristics, such as lower GA, BW and Apgar scores, and were more likely to develop IUGR and fetal distress. Premature infants in group 2 were more likely to develop HsPDA and early PH $(P<0.05)$ (Table 1$)$.

Whether in group 1 or group 2, the level of postnatal NTproBNP showed an upward trend. The NT-proBNP value was at a high level on the 3rd day, and gradually decreased thereafter. On the 7 th day ( \pm 2 days), the median (IQR) NT-proBNP levels of the two groups were 1590 (962-2,708) ng/L and 9185 (4010-23,975) $\mathrm{ng} / \mathrm{L}$ respectively, and gradually tended to a stable low level on the 28th day ( \pm 2 days) (Figure 1). The NT-proBNP levels in group 2 were higher than those in group 1 from day 3 to day 28 ( \pm 2 days) after birth $(p<0.001)$. When the natural logarithm of the serum NT-ProBNP level increased by 1 unit on the 7 th day ( \pm 2 days), the risk of moderate-to-severe $\mathrm{BPD} /$ death was the highest $(\mathrm{OR}=3.753$; 95\%CI: 2.984 4.720; $P<0.001$; Table 2 ), and the predictive ability was the strongest. ROC curve analysis estimated that the AUC value was 0.861 (0.819 0.903), the best cutoff value of NT-proBNP on day 7 after birth ( \pm 2 days) was $3360 \mathrm{ng} / \mathrm{L}$, the sensitivity was $80.0 \%$, and the specificity was $86.2 \%$ (Figure 2).

$\mathrm{MV}$ and respiratory support patterns are risk factors for BPD (23). Consistent with previous studies, patients in group 2 received a longer $\mathrm{MV}$ time in the first week of life and higher respiratory support patterns on the 7 th day ( \pm 2 days) than those in group $1(P<0.001)$. No significant difference was found in the use of caffeine between the groups 1 week after birth $(P>$ 0.05; Table 3). After adjusting the risk factors (GA, BW, 1 - and 5min Apgar scores, history of abnormal pregnancy, fetal distress, IUGR, use of surfactants, HsPDA, early PH, MV time, respiratory support pattern) determined by univariate analysis, the NTproBNP level on the 7 th day ( \pm 2 days) was still an important predictor of moderate-to-severe $\mathrm{BPD} /$ death $(\mathrm{OR}=3.152$; 95\%CI: 2.347 4.234; $P<0.001$; Table 4). The AUCs of the two ROC curves were $0.846(0.809,0.882)$ and $0.906(0.877,0.934)(P<$ $0.001)$, which showed that the level of NT-proBNP on the 7 th day after birth ( \pm 2 days) greatly improved the predictive ability of moderate-to-severe BPD/death (Figure 3).

Based on the multivariate logistic regression and ROC curve results, our study constructed a good model of the early prediction of moderate to severe BPD/death, including the NTproBNP level. To show the model more easily and intuitively, we used six variables with $P<0.05$ to construct a risk prediction nomogram (Figure 4). The calibration curve for the nomogram indicated good consistency in this cohort (Figure 5). 
TABLE 1 | Baseline characteristics and comparison of both study groups.

\begin{tabular}{|c|c|c|c|c|}
\hline & $\begin{array}{l}\text { All patients } \\
(n=556)\end{array}$ & $\begin{array}{l}\text { No-to-mild BPD } \\
\quad(n=418)\end{array}$ & $\begin{array}{l}\text { Moderate-to-severe BPD/death } \\
\qquad(n=138)\end{array}$ & $P$-value \\
\hline GA (weeks) [median (IQR)] & 29.6(28.6,30.5) & $30.0(29.1,31.0)$ & $29.1(28.0,30.0)$ & $<0.001$ \\
\hline Male (\%) & 295(53.0) & $207(49.5)$ & $88(63.8)$ & 0.004 \\
\hline Multiple pregnancy (\%) & $134(24.1)$ & 100(23.9) & $34(24.6)$ & 0.865 \\
\hline Maternal age (years) [median (IQR)] & $30(27,35)$ & $30(27,35)$ & $31(27,36)$ & 0.471 \\
\hline HDCP (\%) & $254(45.6)$ & 193(46.2) & $61(44.2)$ & 0.687 \\
\hline GDM (\%) & $83(14.9)$ & 64(15.3) & 19(13.8) & 0.659 \\
\hline Hypothyroidism with pregnancy (\%) & $30(5.3)$ & $21(5)$ & $9(6.5)$ & 0.5 \\
\hline History of abnormal pregnancy (\%) & 154(27.6) & $106(25.4)$ & 48(34.8) & 0.032 \\
\hline General anesthesia (\%) & $144(25.8)$ & 107(25.6) & $37(26.8)$ & 0.778 \\
\hline PROM (\%) & 136(24.4) & $108(25.8)$ & 28(20.3) & 0.189 \\
\hline Fetal distress (\%) & $120(21.5)$ & $78(18.7)$ & $42(30.4)$ & 0.004 \\
\hline IUGR (\%) & $51(9.1)$ & $28(6.7)$ & 23(16.7) & $<0.001$ \\
\hline Use of surfactant (\%) & $452(81.2)$ & $324(77.5)$ & 128(92.8) & $<0.001$ \\
\hline RDS (\%) & $530(95.3)$ & 397(95) & 133(96.4) & 0.499 \\
\hline HsPDA (\%) & $177(31.8)$ & 112(26.8) & $65(47.1)$ & $<0.001$ \\
\hline Early PH (\%) & $111(20)$ & 75 (17.9) & $36(26.1)$ & 0.038 \\
\hline
\end{tabular}

BPD, bronchopulmonary dysplasia; GA, gestational age; BW, birth weight; IQR, Interquartile range; HDCP, hypertensive disorder complicating pregnancy; GDM, gestation diabetes mellitus; PROM, premature rupture of membranes; IUGR, intrauterine growth restriction; RDS, respiratory distress syndrome; HsPDA, hemodynamically significant patent ductus arteriosus; PH, pulmonary hypertension.
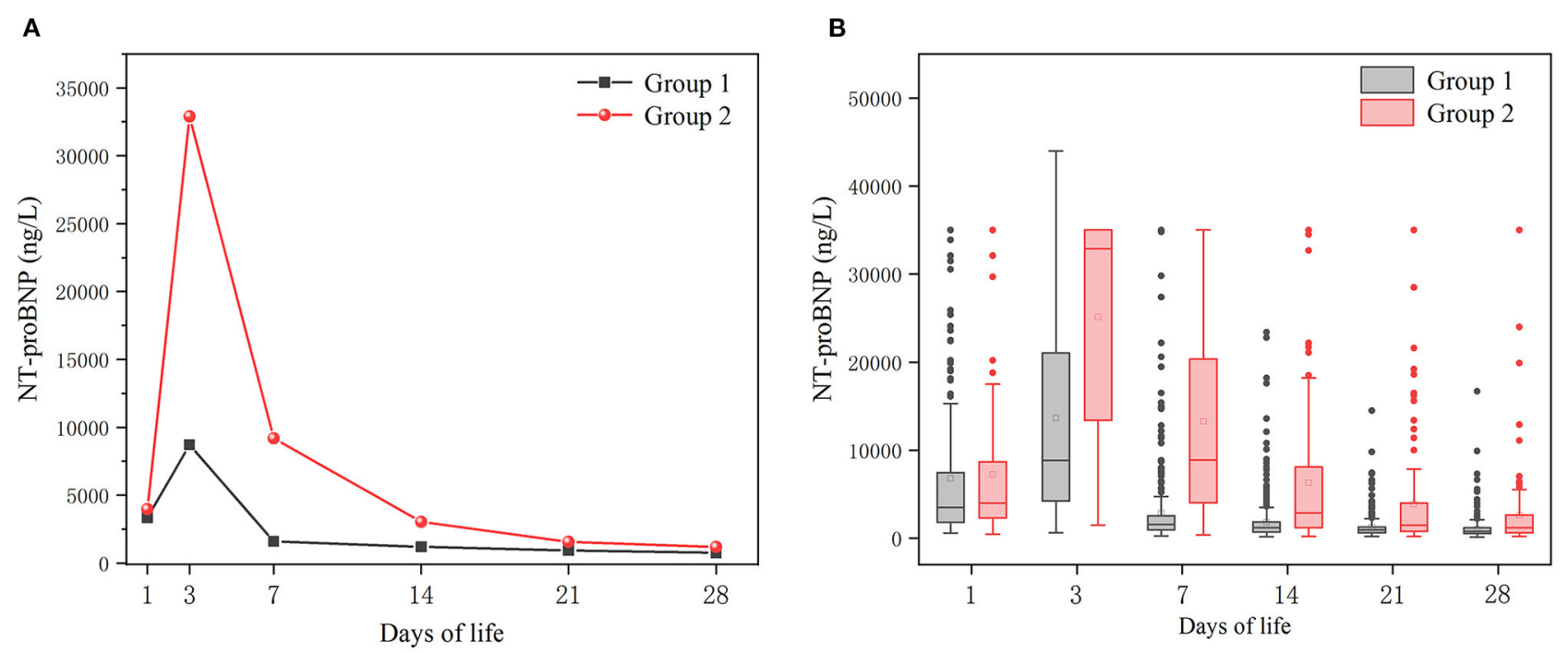

FIGURE 1 | Temporal evolution of NT-proBNP levels in the two groups. Group 1: no-to-mild BPD group; Group 2: moderate-to-severe BPD/death group. (A) Median values of NTproBNP levels. (B) Box plot of NTproBNP levels. 
TABLE 2 | Univariate logistic regression of the association between NT-proBNP values (and IQR) during the first 28 days of life and moderate-to-severe BPD/death.

\begin{tabular}{lccccccc}
\hline Days & Num & All patients & No to mild BPD & Moderate to severe BPD/Death & P-value & OR & 95\% CI \\
\hline 1 & 556 & $8.19(7.59,8.96)$ & $8.15(7.49,8.92)$ & $8.29(7.74,9.09)$ & 0.122 & 1.196 & $(0.990,1.445)$ \\
3 & 556 & $9.40(8.48,10.43)$ & $9.09(8.35,9.96)$ & $10.40(9.48,10.46)$ & $<0.001$ & 2.764 & $(2.137,3.576)$ \\
7 & 556 & $7.59(6.96,8.54)$ & $7.34(6.84,7.85)$ & $9.08(8.30,9.93)$ & $<0.001$ & 3.753 & $(2.984,4.720)$ \\
14 & 531 & $7.13(6.66,7.78)$ & $7.07(6.55,7.52)$ & $7.96(7.08,9.00)$ & $<0.001$ & 2.375 & $(1.926,2.928)$ \\
21 & 511 & $6.88(6.42,7.37)$ & $6.83(6.38,7.15)$ & $7.28(6.63,8.32)$ & $<0.001$ & 2.361 & $(1.860,2.998)$ \\
28 & 476 & $6.75(6.31,7.16)$ & $6.63(6.27,7.07)$ & $7.08(6.38,7.88)$ & $<0.001$ & 2.330 & $(1.768,3.069)$ \\
\hline
\end{tabular}

BPD, bronchopulmonary dysplasia; IQR, Interquartile range; OR, Odds ratio; CI, confidence interval. All NT-proBNP values were converted to logarithm and presented as median (IQR).

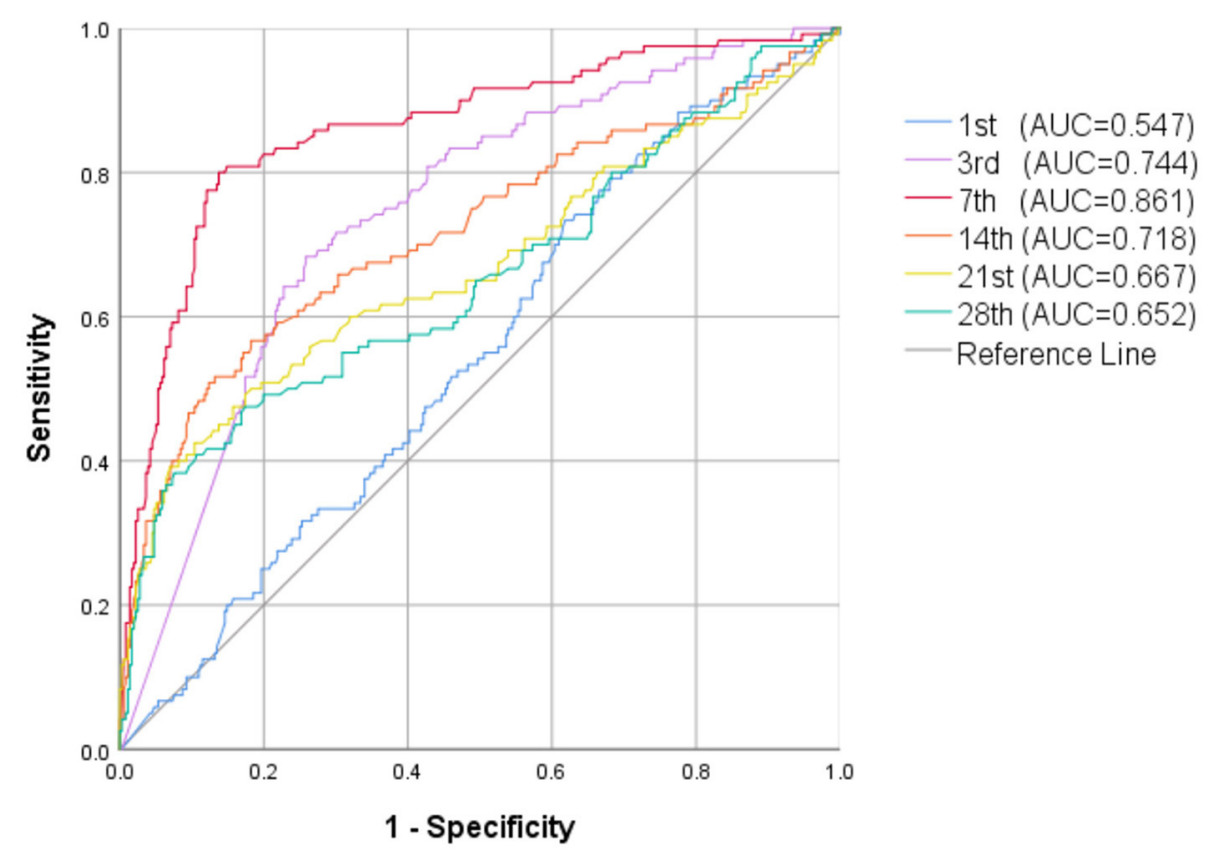

FIGURE 2 | ROC curve of NT-proBNP levels in the first 28 days of life for prediction of moderate-to-severe BPD/death.

TABLE 3 | Comparison of respiratory support and caffeine use in 1 week after birth of both study groups.

\begin{tabular}{|c|c|c|c|c|}
\hline & All patients $(n=556)$ & No-to-mild BPD $(n=418)$ & Moderate-to-severe BPD/death $(n=138)$ & $P$-value \\
\hline Type of respiratory support of Day7 & & & & $<0.001$ \\
\hline Warm box/Low-flow nasal cannula (\%) & $39(7.0)$ & $38(9.1)$ & $1(0.7)$ & \\
\hline NIPPV (\%) & 299(53.8) & $247(59.1)$ & $52(37.7)$ & \\
\hline
\end{tabular}

BPD, bronchopulmonary dysplasia; NIPPV, non-invasive positive pressure ventilation; MV, mechanical ventilation.

\section{DISCUSSION}

To our best knowledge, this study was the first to dynamically evaluate the correlation between the NT-proBNP levels and moderate-to-severe BPD/death in the first 28 days of life, and it had the largest sample size in the study of the correlation between NT-proBNP and BPD. Moderate-to-severe BPD or death was used as the outcome rather than moderate-to-severe BPD because death is a competing outcome with BPD (24). Our results showed that the serum NT-proBNP level of infants 
TABLE 4 | Fully adjusted multivariable estimates of association between NT-proBNP values at day 7 of life and moderate-to-severe BPD/death ${ }^{\text {. }}$

\begin{tabular}{|c|c|c|c|c|}
\hline Predictor & B & OR & $95 \% \mathrm{Cl}$ & $\boldsymbol{P}$ \\
\hline GA & -0.206 & 0.814 & $(0.669,0.990)$ & 0.04 \\
\hline Male & 0.766 & 2.172 & $(1.257,3.753)$ & 0.005 \\
\hline IUGR & 1.329 & 3.776 & $(1.603,8.899)$ & 0.002 \\
\hline NT-proBNP7 & 1.148 & 3.152 & $(2.347,4.234)$ & $<0.001$ \\
\hline Days of MV, 1st week & -0.372 & 0.690 & $(0.567,0.839)$ & $<0.001$ \\
\hline Type of respiratory support of day 7 & 2.551 & 12.817 & $(4.325,37.989)$ & $<0.001$ \\
\hline
\end{tabular}

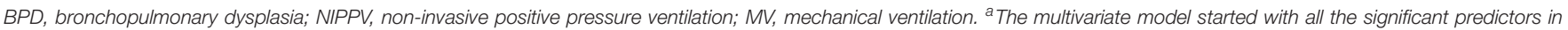
Table 1 and Table 3. The model went through the stepwise selection by keeping only the statistical significant variables in the final multivariate model.

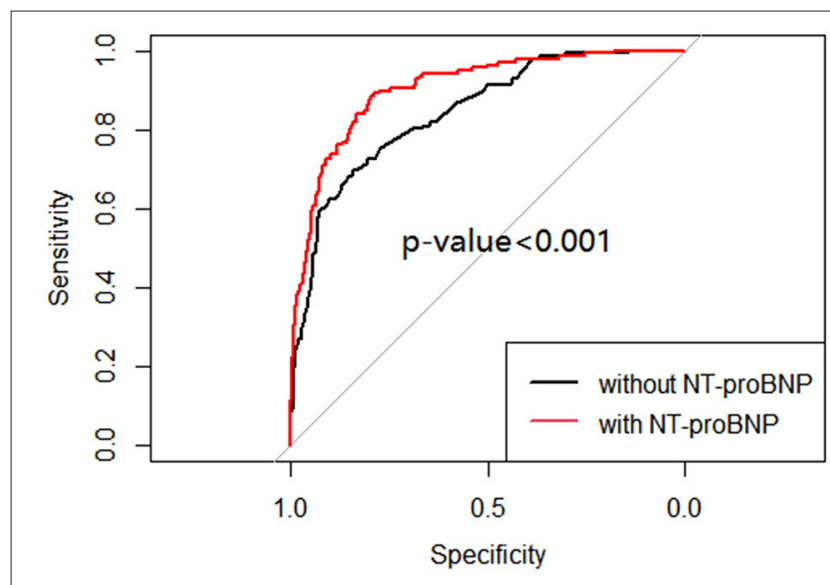

FIGURE 3 | ROC curve of multivariable logistic regression of moderate-to-severe BPD/death with and without NT-proBNP level.

with moderate-to-severe $\mathrm{BPD} /$ death was significantly higher than that of infants without/mild BPD from 3 to 28 days after birth, and the predictive value on the 7 th day after birth ( \pm 2 days) was the highest (the cutoff value was $3360 \mathrm{ng} / \mathrm{L}$, the sensitivity was $80.0 \%$, and the specificity was $86.2 \%$ ) in VLBWs. Moreover, it remained statistically significant after controlling for confounding variables, including GA and other factors. We also developed a simple and convenient nomogram to predict moderate-to-severe $\mathrm{BPD} /$ death that helped clinicians make better treatment decisions and increased the possibility of early intervention for high-risk patients.

In the past few decades, many predictive models for BPD have been developed using clinical variables, such as GA, BW, sex, IUGR, MV time, and HsPDA $(25,26)$. As expected, smaller GA, male sex, IUGR, MV time in the first week of life and type of respiratory support on the 7 th day after birth were important predictors of moderate-to-severe $\mathrm{BPD} / \mathrm{death}$, and patients with HsPDA were more likely to develop moderate-to-severe BPD. These known risk factors have increased neonatal pediatricians' awareness of the potential risk of BPD in preterm infants, but the recognition of high-risk patients with moderate-to-severe BPD was limited, making early intervention difficult for specific patients who are most likely to develop the most severe BPD grade and indicating the need for more reliable parameters to improve the accuracy of prediction. Increasing evidence supports the use of the NT-proBNP levels as a biomarker for screening, diagnosis, management and prognosis of heart disease in children $(27,28)$. Consistent with Kulkarni et al.'s study (29), NT-proBNP levels increased mostly on the 3rd day after birth in our study, because the transition from fetal circulation to neonatal circulation in the first few days of life is accompanied by an increase in pulmonary blood flow and systemic vascular resistance caused by pulmonary dilatation, and a rapid increase in the NT-proBNP levels can reduce the increase in left ventricular load and support myocardial function $(30,31)$. The serum NTproBNP concentration of healthy newborns gradually stabilizes in the third month after birth (32). If neonatal diseases such as HsPDA (33), congestive heart defects (34), diaphragmatic hernia (35) and anemia (36) occur, the NT-proBNP level will remain at a high level.

Many studies have used the serum NT-proBNP levels as a potential biomarker of respiratory diseases, including $\mathrm{BPD}$ in premature infants $(37,38)$. However, the mechanism of the increase in NT-proBNP in BPD is not completely clear. In adult studies, plasma NT-proBNP can be used as a biomarker of the exacerbation of chronic obstructive pulmonary disease (39). Our previous studies have shown that NTproBNP can be used to predict ventilator weaning failure in premature infants with RDS, and a high level of NTproBNP corresponds to a high rate of extubation failure (40), increasing the risk of BPD (41). The changes in the plasma NTproBNP levels suggest changes in intrauterine and extrauterine hemodynamics in children. The increase in the plasma NTproBNP levels in patients with BPD may be related to increased pulmonary vascular pressure, chronic lung disease, diastolic dysfunction or impaired left ventricular function (42). The development of BPD destroys alveolarization and microvascular development, which increases the resistance of pulmonary vessels and may lead to the occurrence of BPD-related $\mathrm{PH}$ $(43,44)$. In premature infants, excessive pulmonary pressure in the immature lungs is accompanied by the continuous maturation of the alveolar and vascular structure, leading to the abnormal development of pulmonary blood vessels (45). At the same time, the response of pulmonary vessels 


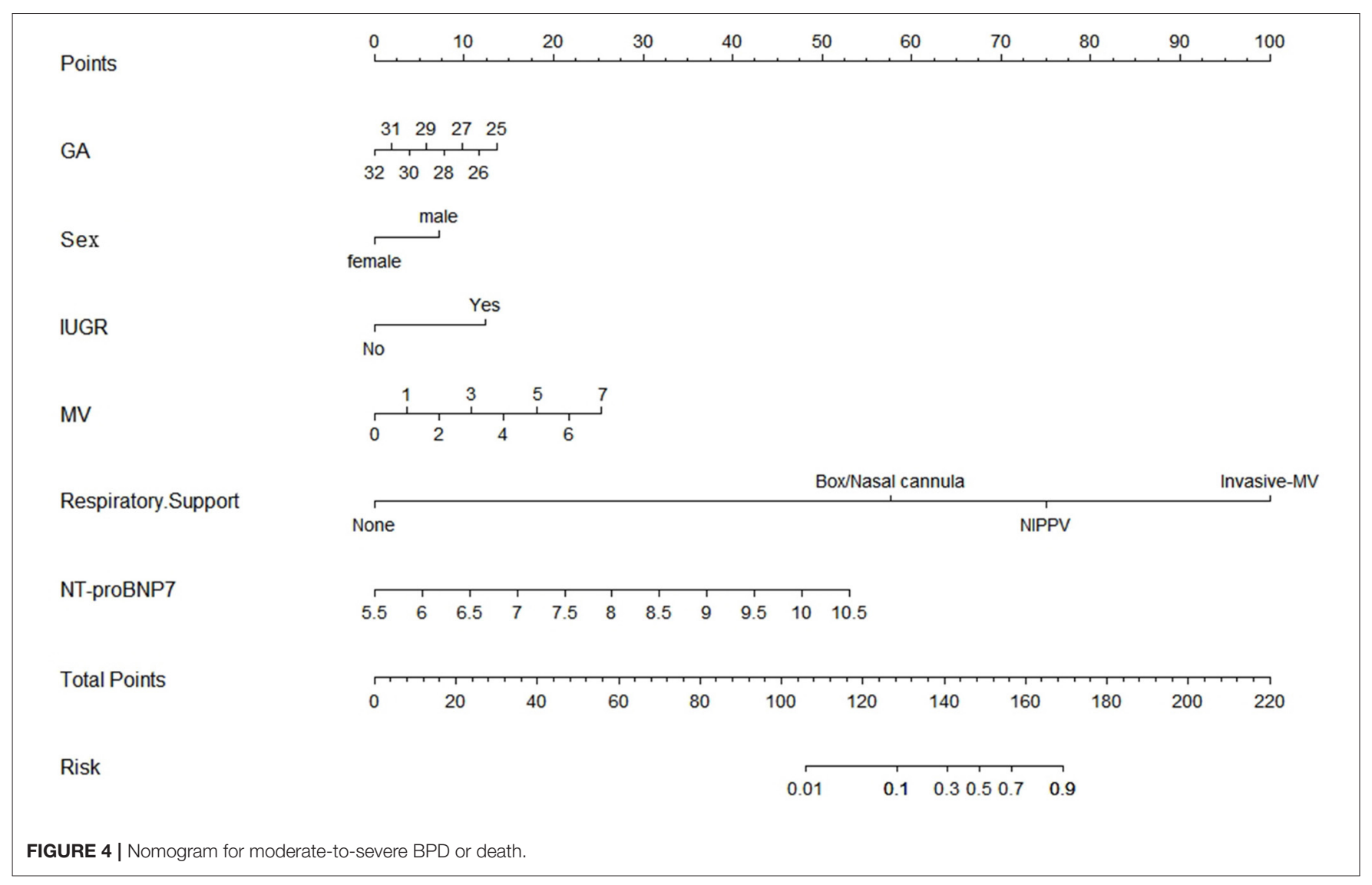

to oxygen may be enhanced, and mild hypoxia can also lead to a significant increase in pulmonary artery pressure (46). Many studies have shown that children with $\mathrm{PH}$ have higher levels of NT-proBNP (47-50). In addition to postnatal factors, experimental studies support epidemiological findings that prenatal causes not only short-term abnormalities in respiratory function, but also persistent destruction of the lung and pulmonary vascular structure throughout infancy (such as IUGR). Thus, adverse intrauterine stimulation is sufficient to damage vascular growth and induce long-term and severe $\mathrm{PH}(51,52)$.

A recent prospective study of 229 premature infants suggested that the precursors of atrial natriuretic peptide (ANP) levels measured on the 7 th day of life ( \pm 2 days) were associated with combined outcomes of BPD or death in univariable models but not after adjusting for cofactors (53). Both ANP and BNP belong to the natriuretic peptide family. ANP is released from atrial myocytes in response to atrial wall stretching and is mostly collinear in children and adults (54). Although ANP is unstable, its secretion can be estimated by measuring the mid-regional proatrial natriuretic peptide (MR-proANP). Thus, future studies can add valuable information concerning MR-proANP as a predictor of the occurrence and severity of BPD.

Our study has several limitations. Our data were collected at a single center and may not represent all BPD patients.
Additionally, we excluded patients with hereditary metabolic disorders, multiple malformations, severe congenital heart disease and renal insufficiency. Thus this study could not be extended to these specific populations. To measure NTproBNP, the NT-proBNP value higher than the quantitative upper limit $(>35,000 \mathrm{ng} / \mathrm{L})$ was changed to 35,010 , but the real value may be much higher than this value, producing a higher average value of NT-proBNP than that calculated in this study. Additionally, this is a relatively mature cohort for BPD, and the results may be different in infants with a smaller GA. In the future, a prospective study involving a larger sample size, multicenter, and internal and external validation is needed to incorporate NT-proBNP measurements into the study of predicting the outcome of preterm infants.

\section{CONCLUSION}

The level of NT-proBNP on the 7th day after birth ( \pm 2 days) can be used as a valuable early biomarker for predicting moderate and severe BPD/death, and the established nomogram can help clinicians predict the risk effectively and easily. Our findings warrant a larger prospective study to include NT-proBNP measurements in predicting the outcomes of VLBWs. 


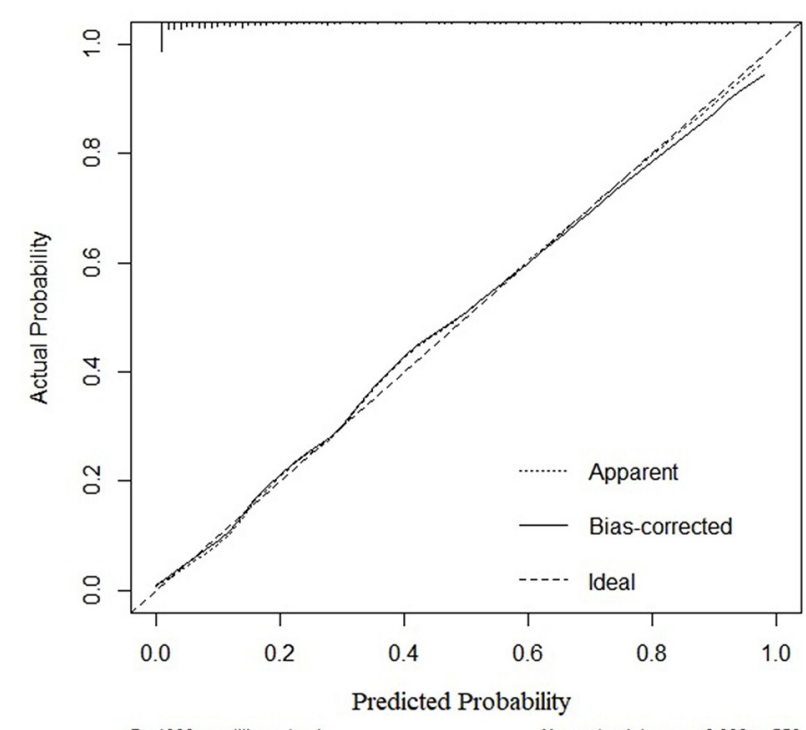

FIGURE 5 | Calibration curves for the nomogram of moderate-to-severe BPD or death.

\section{DATA AVAILABILITY STATEMENT}

The raw data supporting the conclusions of this article will be made available by the authors, without undue reservation.

\section{ETHICS STATEMENT}

The studies involving human participants were reviewed and approved by the Medical Ethics Committee of the First Affiliated

\section{REFERENCES}

1. Stoll BJ, Hansen NI, Bell EF, Shankaran S, Laptook AR, Walsh $\mathrm{MC}$, et al. Neonatal outcomes of extremely preterm infants from the NICHD Neonatal Research Network. Pediatrics. (2010) 126:443-56. doi: 10.1542/peds.2009-2959

2. Thébaud B, Goss KN, Laughon M, Whitsett JA, Abman SH, Steinhorn RH, et al. Bronchopulmonary dysplasia. Nat Rev Dis Primers. (2019) 5:123. doi: 10.1038/s41572-019-0127-7

3. Gomez Pomar E, Concina VA, Samide A, Westgate PM, Bada HS. Bronchopulmonary dysplasia: comparison between the two most used diagnostic criteria. Front Pediatr. (2018) 6:397. doi: 10.3389/fped.2018.00397

4. Isayama T, Lee SK, Yang J, Lee D, Daspal S, Dunn M, et al. Revisiting the definition of bronchopulmonary dysplasia: effect of changing panoply of respiratory support for preterm neonates. JAMA Pediatr. (2017) 171:2719. doi: 10.1001/jamapediatrics.2016.4141

5. Abman SH, Bancalari E, Jobe A. The evolution of bronchopulmonary dysplasia after 50 years. Am J Respir Crit Care Med. (2017) 4214. doi: 10.1164/rccm.201611-2386ED

6. Sharma A, Xin Y, Chen X, Sood SG. Early prediction of moderate to severe bronchopulmonary dysplasia in extremely premature infants. Pediatr Neonatol. (2020) 61:290-9. doi: 10.1016/j.pedneo.2019.12.001

7. Stensvold HJ, Klingenberg C, Stoen R, Moster D, Braekke K, Guthe HJ, et al. Neonatal morbidity and 1-year survival of extremely preterm infants. Pediatrics. (2017) 139:e20161821. doi: 10.1542/peds.2016-1821
Hospital of Zhengzhou University (Ethical code: 2019-KY-95). Written informed consent to participate in this study was provided by the participants' legal guardian/next of kin.

\section{AUTHOR CONTRIBUTIONS}

QZ, MS, and ML conceptualized and designed the study, drafted the initial manuscript, reviewed, and revised the manuscript. WD, CL, ZS, and XC designed the data collection instruments, collected data, carried out the initial analyses, and revised the manuscript. LW, FM, PX, and MW coordinated and supervised data collection and critically reviewed the manuscript for important intellectual content. WC, JG, and JZ designed the tables and figures of this article. QZ made a great contribution to the design and implementation of the whole study. All authors approved the final manuscript as submitted and agree to be accountable for all aspects of the work.

\section{FUNDING}

All phases of this study were supported by Joint Construction Project of Medical Science and Technology in Henan Province, No. LHGJ20190064 (to ML), National Health Commission Medical and Health Science and Technology Development Center, No. WA2020HK41 (to QZ) and Science and Technology Department of Henan Province project, No. 172102410017 (to QZ).

\section{ACKNOWLEDGMENTS}

We thank The First Affiliated Hospital of Zhengzhou University, Translational Medical Center for its support and assistance throughout the research process.

8. Jensen EA, Dysart K, Gantz MG, McDonald S, Bamat NA, Keszler M, et al. The diagnosis of bronchopulmonary dysplasia in very preterm infants. An evidence-based approach. Am J Resp Crit Care. (2019) 200:7519. doi: $10.1164 / \mathrm{rccm} .201812-2348 \mathrm{OC}$

9. Weber M, Hamm C. Role of B-type natriuretic peptide (BNP) and NT-proBNP in clinical routine. Heart. (2006) 92:8439. doi: 10.1136/hrt.2005.071233

10. Fritz AS, Keller T, Kribs A, Hünseler C. Reference values for n-terminal probrain natriuretic peptide in premature infants during their first weeks of life. Eur J Pediatr. (2021) 180:1-9. doi: 10.1007/s00431-020-03853-8

11. Sellmer A, Hjortdal VE, Vibeke, Bjerre JV, Schmidt MR, McNamara PJ, et al. $\mathrm{N}$-terminal pro-b type natriuretic peptide as a marker of bronchopulmonary dysplasia or death in very preterm neonates: a cohort study. PLOS ONE. (2015) 10:e0140079. doi: 10.1371/journal.pone.0140079

12. Harris SL, More K, Dixon B, Troughton R, Pemberton C, Horwood $\mathrm{J}$, et al. Factors affecting N-terminal pro-B-type natriuretic peptide levels in preterm infants and use in determination of haemodynamic significance of patent ductus arteriosus. Eur J Pediatr. (2018) 177:52132. doi: 10.1007/s00431-018-3089-y

13. Khan S, Concina VA, Schneider D, Westgate P, Arriagada S, Bada H. Role of NT-proBNP in the prediction of moderate to severe Bronchopulmonary Dysplasia in preterm infants. Pediatr Pulmonol. (2020) 55:376-82. doi: 10.1002/ppul.24610

14. Jobe AH, Bancalari E. Bronchopulmonary dysplasia. Am J Respir Crit Care Med. (2001) 163:1723-9. doi: 10.1164/ajrccm.163.7.2011060 
15. Alves E, Azevedo A, Rodrigues T, Santos AC, Barros H. Impact of risk factors on hypertensive disorders in pregnancy, in primiparae and multiparae. Ann Hum Biol. (2013) 40:377-84 doi: 10.3109/03014460.2013.793390

16. Alexander EK, Pearce EN, Brent GA, Brown RS, Chen H, Dosiou C, et al. 2017 guidelines of the American thyroid association for the diagnosis and management of thyroid disease during pregnancy and the postpartum. Thyroid. (2017) 27:315-89. doi: 10.1089/thy.2016.0457

17. Elimian A, Lawlor P, Figueroa R, Wiencek V, Garry D, Quirk JG. Intrapartum assessment of fetal well-being: any role for a fetal admission test? J Matern Fetal Neonatal Med. (2003) 13:408-13. doi: 10.1080/jmf.13.6.408.413

18. ACOG Practice Bulletin Number 227: Fetal Growth Restriction. Obstet Gynecol. (2021) 137: e16-e28. doi: 10.1097/AOG.0000000000004251

19. Li Y, Zhang C, Zhang D. Cesarean section and the risk of neonatal respiratory distress syndrome: a meta-analysis. Arch Gynecol Obstet. (2019) 300:50317. doi: 10.1007/s00404-019-05208-7

20. Sung SI, Yun SC, Ji YC, Yoon SA, Park WS. Mandatory closure versus nonintervention for patent ductus arteriosus in very preterm infants. J Pediatr. (2016) 177:66-71. doi: 10.1016/j.jpeds.2016.06.046

21. Skinner JR, Boys RJ, Hunter S, Hey EN. Non-invasive assessment of pulmonary arterial pressure in healthy neonates. Arch Dis Child. (1991) 66 (4 Spec No):386-90. doi: 10.1136/adc.66.4_Spec_No.386

22. Harrell FE. Regression Modeling Strategies: With Applications to Linear Models, Logistic and Ordinal Regression, and Survival Analysis. New York, NY: Springer-Verlag (2015).

23. Laughon MM, Langer JC, Bose CL, Smith PB, Ambalavanan N, Kennedy $\mathrm{KA}$, et al. Prediction of bronchopulmonary dysplasia by postnatal age in extremely premature infants. Am J Respir Crit Care Med. (2011) 183:171522. doi: 10.1164/rccm.201101-0055OC

24. Andersen PK, Geskus RB, de Witte T, Putter H. Competing risks in epidemiology: possibilities and pitfalls. Int J Epidemiol. (2012) 41:86170. doi: 10.1093/ije/dyr213

25. Zhang J, Luo C, Lei M, Shi Z, Cheng X, Wang L, et al. Development and validation of a nomogram for predicting bronchopulmonary dysplasia in very-low-birth-weight infants. Front Pediatr. (2021) 9:200. doi: 10.3389/fped.2021.648828

26. Nino G, Mansoor A, Perez GF, Arroyo M, Xuchen X, Weinstock $\mathrm{J}$, et al. Validation of a new predictive model to improve risk stratification in bronchopulmonary dysplasia. Sci Rep. (2020) 10:613. doi: 10.1038/s41598-019-56355-5

27. Hauser JA, Demyanets S, Rusai K, Goritschan C, Weber M, Panesar D, et al. Diagnostic performance and reference values of novel biomarkers of paediatric heart failure. Heart. (2016) 102:1633-9. doi: 10.1136/heartjnl-2016-309460

28. Cantinotti M, Walters HL, Crocetti M, Marotta M, Murzi B, Clerico A. BNP in children with congenital cardiac disease: is there now sufficient evidence for its routine use? Cardiol Young. (2015) 25:42437. doi: 10.1017/S1047951114002133

29. Kulkarni M, Gokulakrishnan G, Price J, Fernandes CJ, Leeflang M, Pammi M. Diagnosing significant PDA using natriuretic peptides in preterm neonates: a systematic review. Pediatrics. (2015) 135:e51025. doi: 10.1542/peds.2014-1995

30. Mir TS, Laux R, Hellwege HH, Liedke B, Heinze C, Buelow HV, et al. Plasma concentrations of aminoterminal pro atrial natriuretic peptide and aminoterminal pro brain natriuretic peptide in healthy neonates: marked and rapid increase after birth. Pediatrics. (2003) 112:8969. doi: 10.1542 /peds.112.4.896

31. da Graca RL, Hassinger DC, Flynn PA, Sison CP, Nesin M, Auld PAM. Longitudinal changes of brain-type natriuretic peptide in preterm neonates. Pediatrics. (2006) 117:2183-9. doi: 10.1542/peds.2005-1387

32. Lechner E, Wiesinger-Eidenberger G, Wagner O, Weissensteiner M, SchreierLechner E, Leibetseder D, et al. Amino terminal pro B-type natriuretic peptide levels are elevated in the cord blood of neonates with congenital heart defect. Pediatr Res. (2009) 66:466-9. doi: 10.1203/PDR.0b013e3181 b3aee 4

33. Letshwiti JB, Sirc J, RO'Kelly, Miletin J. Serial n-terminal pro-brain natriuretic peptide measurement as a predictor of significant patent ductus arteriosus in preterm infants beyond the first week of life. Eur J Pediatr. (2014) 173:14916. doi: 10.1007/s00431-014-2350-2
34. Cantinotti M, Law Y, Vittorini S, Crocetti M, Marco M, Murzi B, et al. The potential and limitations of plasma BNP measurement in the diagnosis, prognosis, and management of children with heart failure due to congenital cardiac disease: an update. Heart Fail Rev. (2014) 19:72742. doi: 10.1007/s10741-014-9422-2

35. Snoek KG, Kraemer US, Ten Kate CA, Greenough A, Van Heijst A, Capolupo $\mathrm{I}$, et al. High-sensitivity troponin $\mathrm{T}$ and $\mathrm{N}$-terminal pro-brain natriuretic peptide in prediction of outcome in congenital diaphragmatic hernia: results from a multicenter, randomized controlled trial. J Pediatr. (2016) 173:24549. doi: 10.1016/j.jpeds.2016.03.026

36. Duan J, Kong X, Li Q, Hua S, Zhang S, Zhang X, et al. Association between anemia and bronchopulmonary dysplasia in preterm infants. Sci Rep. (2016) 6:1-6. doi: 10.1038/srep22717

37. Sztefko K. NTproBNP: a biomarker with new potential application. Pol Arch Med Wewn. (2015) 125:509-10. doi: 10.20452/pamw.2989

38. Zhou L, Xiang X, Wang L, Chen X, Zhu J, Xia H. N-Terminal Pro-B-type natriuretic peptide as a biomarker of bronchopulmonary dysplasia or death in preterm infants: a retrospective cohort analysis. Front Pediatr. (2019) 7:166. doi: $10.3389 /$ fped.2019.00166

39. Shafuddin E, Fairweather SM, Chang CL, Tuffery C, Hancoxet RJ. Cardiac biomarkers and long-term outcomes of exacerbations of COPD: a long-term follow-up of two cohorts. ERJ Open Res. (2021) 7. doi: 10.1183/23120541.00531-2020

40. Zhang Q, Shi ZY, Luo CH, Wang L, Zhang SS, Cheng XR, et al. Application of NT-proBNP in ventilator weaning for preterm infants with RDS. Pediatr Pulmonol. (2014) 49:757-63. doi: 10.1002/ppul.22875

41. Shalish W, Kanbar L, Kovacs L, Chawla S, Keszler M, Rao S, et al. The impact of time interval between extubation and reintubation on death or bronchopulmonary dysplasia in extremely preterm infants. J Pediatr. (2019) 205:70-6. doi: 10.1016/j.jpeds.2018.09.062

42. Vijlbrief DC, Benders MJNL, Kemperman H, van Bel F, de Vries WB. B-type natriuretic peptide and rebound during treatment for persistent pulmonary hypertension. J Pediatr. (2012) 160:111-5. doi: 10.1016/j.jpeds.2011. 06.038

43. Jobe AH. The new bronchopulmonary dysplasia. Curr Opin Pediatr. (2011) 23:167-72. doi: 10.1097/MOP.0b013e3283423e6b

44. König K, Guy KJ, Nold-Petry CA, Barfield CP, Walsh G, Drew SM, et al. BNP, troponin I, and YKL-40 as screening markers in extremely preterm infants at risk for pulmonary hypertension associated with bronchopulmonary dysplasia. Am J Physiol Lung Cell Mol Physiol. (2016) 311:L1076-81. doi: 10.1152/ajplung.00344.2016

45. Mata-Greenwood E, Meyrick B, Soifer SJ, Fineman JR, Black SM. Expression of VEGF and its receptors Flt-1 and Flk-1/KDR is altered in lambs with increased pulmonary blood flow and pulmonary hypertension. Am J Physiol Lung Cell Mol Physiol. (2003) 285:222-31. doi: 10.1152/ajplung.0038 8.2002

46. Mourani PM, Ivy DD, Gao D, Abman SH. Pulmonary vascular effects of inhaled nitric oxide and oxygen tension in bronchopulmonary dysplasia. Am J Respir Crit Care Med. (2004) 170:1006-13. doi: 10.1164/rccm.2003101483OC

47. Montgomery AM, Bazzy-Asaad A, Asnes JD, Bizzarro MJ, Ehrenkranz RA, Weismann CG. Biochemical screening for pulmonary hypertension in preterm infants with bronchopulmonary dysplasia. Neonatology. (2016) 109:190-4. doi: 10.1159/000442043

48. Xiong T, Kulkarni M, Gokulakrishnan G, Shivanna B, Pammi M. Natriuretic peptides in bronchopulmonary dysplasia: a systematic review. J Perinatol. (2020) 40:607-15. doi: 10.1038/s41372-019-0588-2

49. Dasgupta S, Aly AM, Malloy MH, Okorodudu AO, Jain SK. NTproBNP as a surrogate biomarker for early screening of pulmonary hypertension in preterm infants with bronchopulmonary dysplasia. J Perinatol. (2018) 38:1252-7. doi: 10.1038/s41372-018-0164-1

50. König K, Guy KJ, Walsh G, Drew SM, Barfield CP. Association of BNP, NTproBNP, and early postnatal pulmonary hypertension in very preterm infants. Pediatr Pulmonol. (2016) 51:820-4. doi: 10.1002/ppul. 23391

51. Taglauer E, Abman SH, Keller RL. Recent advances in antenatal factors predisposing to bronchopulmonary dysplasia. Semin Perinatol. (2018) 42:413-424. doi: 10.1053/j.semperi.2018.09.002 
52. Mandell EW, Abman SH. Fetal vascular origins of bronchopulmonary dysplasia. $\quad J$ Pediatr. (2017) 185:7-10. doi: 10.1016/j.jpeds.2017. 03.024

53. Gerull R, Neumann RP, Atkinson A, Bernasconi L, Schulzke SM, Wellmann S. Respiratory morbidity in preterm infants predicted by natriuretic peptide (MR-proANP) and endothelin-1 (CT-proET-1). Pediatr Res. (2021) 21:17. doi: $10.1038 / \mathrm{s} 41390-021-01493-8$

54. Song $\mathrm{W}$, Wang $\mathrm{H}, \mathrm{Wu}$ Q. Atrial natriuretic peptide in cardiovascular biology and disease (NPPA). Gene. (2015) 569:1-6. doi: 10.1016/j.gene.2015.06.029

Conflict of Interest: The authors declare that the research was conducted in the absence of any commercial or financial relationships that could be construed as a potential conflict of interest.
Publisher's Note: All claims expressed in this article are solely those of the authors and do not necessarily represent those of their affiliated organizations, or those of the publisher, the editors and the reviewers. Any product that may be evaluated in this article, or claim that may be made by its manufacturer, is not guaranteed or endorsed by the publisher.

Copyright (๑) 2021 Song, Lei, Luo, Shi, Cheng, Ding, Cao, Zhang, Ge, Wang, Xia, Mao, Wang and Zhang. This is an open-access article distributed under the terms of the Creative Commons Attribution License (CC BY). The use, distribution or reproduction in other forums is permitted, provided the original author(s) and the copyright owner(s) are credited and that the original publication in this journal is cited, in accordance with accepted academic practice. No use, distribution or reproduction is permitted which does not comply with these terms. 